\title{
Mammaplasty using an inverted-T technique following the excision of a giant phyllodes tumor
}

\author{
Kazuyuki Kubo $\cdot$ Yuji Hayashi $\cdot$ Hiroyuki Takei $\cdot$ Takashi Yoshida \\ Hiroshi Matsumoto · Sasagu Kurozumi • Masafumi Kurosumi · Hanako Oba • \\ Takashi Saito · Atsumori Hamahata $\cdot$ Hiroyuki Sakurai
}

Received: 20 August 2012/Accepted: 11 January 2013/Published online: 5 February 2013

(C) The Japan Society of Clinical Oncology 2013

\begin{abstract}
We report the case of a woman with a giant phyllodes tumor who successfully underwent a mammaplasty after breast-conserving surgery (BCS). The patient was a 40 -year-old woman with a 25 -cm lump in the left breast. The lesion was diagnosed as a phyllodes tumor by core needle biopsy. The patient was informed about the risks and benefits of BCS and total mastectomy. She selected BCS, and a mammaplasty using an inverted-T technique was performed after the complete resection of the tumor. The skin was expanded, and the nipple-areola was transplanted as a free graft to achieve breast symmetry. The mass was histopathologically diagnosed as a benign phyllodes tumor. The patient was satisfied with the cosmetic results. This method is considered to be one of the options for the treatment of giant phyllodes tumors.
\end{abstract}

Keywords Giant phyllodes tumor - Breast - Oncoplastic surgery $\cdot$ Mastopexy $\cdot$ Reduction mammaplasty

K. Kubo $(\bowtie) \cdot$ Y. Hayashi $\cdot$ H. Takei $\cdot$ T. Yoshida $\cdot$

H. Matsumoto $\cdot$ S. Kurozumi

Division of Breast Surgery, Saitama Cancer Center,

818 Komuro, Ina, Kita-Adachi, Saitama 362-0806, Japan

e-mail: kazu-k@cancer-c.pref.saitama.jp

M. Kurosumi · H. Oba

Department of Pathology, Saitama Cancer Center,

818 Komuro, Ina, Kita-Adachi, Saitama 362-0806, Japan

T. Saito $\cdot$ A. Hamahata

Department of Plastic Surgery, Saitama Cancer Center,

818 Komuro, Ina, Kita-Adachi, Saitama 362-0806, Japan

H. Sakurai

Department of Plastic Surgery, Tokyo Women's Medical

University, 8-1 Kawadacho, Shinjuku-ku,

Tokyo 162-8666, Japan

\section{Introduction}

Oncoplastic surgery is now common in breast cancer treatment [1]. In Japan, post-mastectomy breast reconstruction is now increasingly performed in women with breast cancer who are not candidates for breast-conserving surgery (BCS). This trend has been supported by the national medical insurance, which began covering $70 \%$ of the total cost of breast reconstruction using an autologous tissue flap. Oncoplastic surgery is also used for women with benign breast disorders such as fibroadenomas or phyllodes tumors [2-5]. Oncoplastic surgery is of great importance for breast reconstruction after phyllodes tumor resection because these tumors may grow rapidly and form large masses. In such cases, the tumor removal results in a deformity of the breast that may require a total mastectomy.

We report a case of mammaplasty using an inverted-T technique after the removal of a giant phyllodes tumor of the breast.

\section{Case report}

A 40-year-old woman came to our outpatient clinic because of a large mass in her left breast. She had noticed the mass 2 years prior, and it had rapidly increased in size. The physical examination revealed a mass with a maximum diameter of $25 \mathrm{~cm}$ that occupied the whole left breast and expanded to both the overlying skin and the nipple areolar complex (Fig. 1). Ultrasonography revealed that the mass was lobulated and well circumscribed without invasion of the skin or the chest wall (Fig. 2). The mass was diagnosed as a phyllodes tumor by a core needle biopsy. 


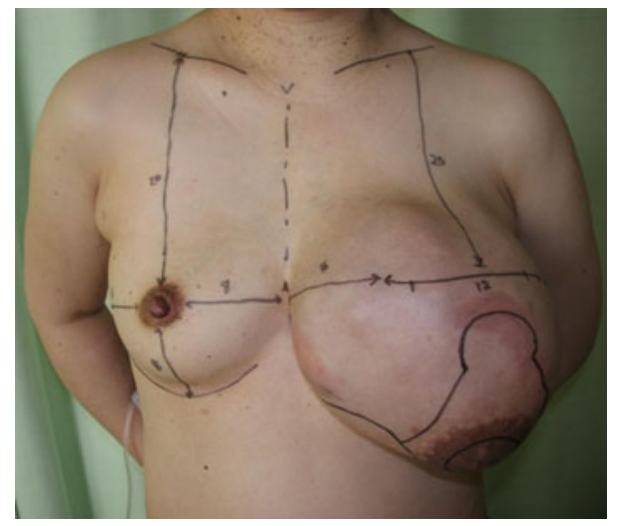

Fig. 1 The preoperative appearance of both breasts. The left breast was hugely enlarged by the giant mass. The skin incisions were aligned in an upright position

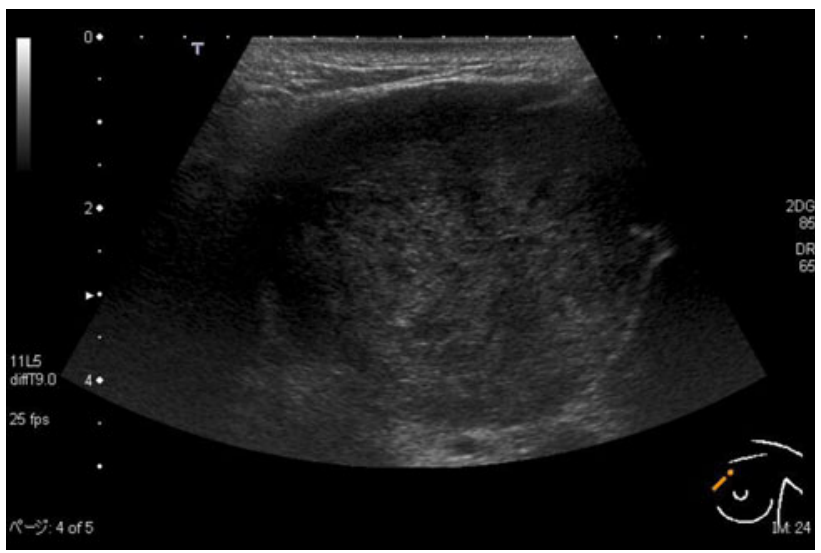

Fig. 2 Ultrasonography revealed that the mass did not invade into the breast skin or the chest wall

We considered two surgical options for this patient: BCS and total mastectomy. The former surgery could include an increased risk of local recurrence if the surgical margins were positive. Although the tumor was close to the overlying skin and nipple-areola complex, no direct invasion to these tissues was confirmed by physical examination or ultrasound. Therefore, we speculated that this tumor could be removed with negative surgical margins in which no exposure of tumor cells would be detected. The latter surgery may reduce the risk of local recurrence, but requires breast reconstruction to avoid cosmetic morbidity; therefore, this was thought to be a more invasive option. The patient was informed about the risks and benefits of both BCS and mastectomy. After careful discussion, she chose BCS. We anticipated that BCS would result in a deformity of the breast, and a mammaplasty with an inverted-T technique was planned.

The skin incisions were planned to reduce the area of expanded skin and to move the nipple-areola complex to the same position as on the contralateral breast. The area of the breast skin identified for removal had a maximal width of
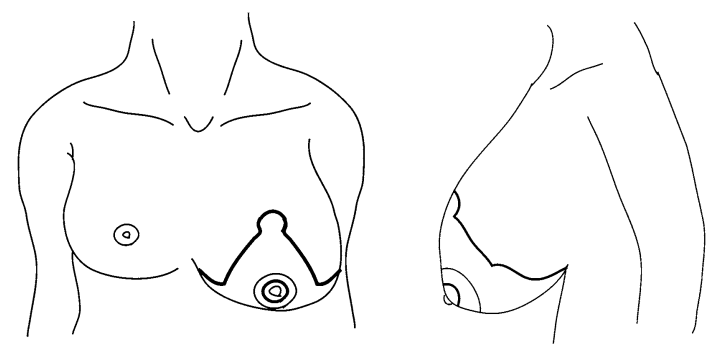

Fig. 3 Schemas of skin incision lines. It was planned that the breast skin would be resected with a maximal width of $10 \mathrm{~cm}$ and that the nipple-areola complex would be reduced in size to a maximal diameter of $2 \mathrm{~cm}$ to be symmetrical with the contralateral breast

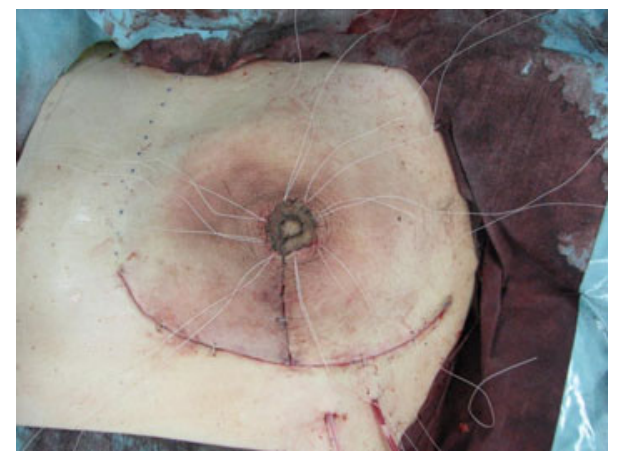

Fig. 4 The postoperative appearance of the left breast. The nippleareola complex was transplanted as a free graft because a glandular flap could not be constructed

$10 \mathrm{~cm}$ horizontally. It was planned that the extended nippleareola complex would be reduced in size to $2-\mathrm{cm}$ diameter to be symmetrical with the contralateral one, and it was to be moved with a glandular flap to the same position as the contralateral nipple-areola complex (Fig. 3).

Under general anesthesia, an infra-mammary incision was made. The mass was fully encapsulated, and it was completely removed without damaging the capsule. Most of the normal glandular tissues were displaced by the tumor to the outer area of the breast, and a glandular flap could not be constructed below the nipple-areola. Therefore, the nipple-areola complex was transplanted as a free graft (Fig. 4).

The macroscopic appearance of the removed tumor is shown in Fig. 5. The tumor was lobulated and encapsulated (Fig. 5a). The maximal cut surface of the tumor was orange colored, solid, and homogeneous (Fig. 5b). The weight of the tumor was $1,442 \mathrm{~g}$. Histopathologically, the tumor consisted of benign epithelial and stromal cells with no obvious mitosis or cellular atypia (Fig. 6). The tumor was histopathologically diagnosed as a benign phyllodes tumor.

Six months postoperatively, the symmetry of the bilateral breast has been almost achieved in terms of the nippleareola complex's position and breast shape. Also, the 
Fig. 5 The macroscopic appearance of the removed tumor. The tumor was lobulated and encapsulated (a). The maximal cut surface of the tumor was orange-colored, solid, and homogeneous (b)
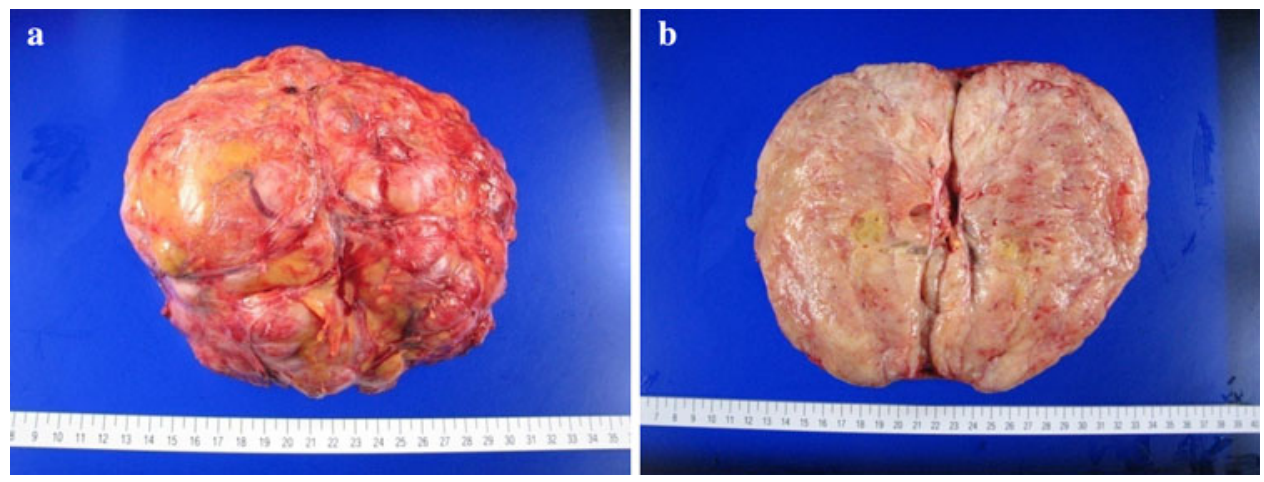

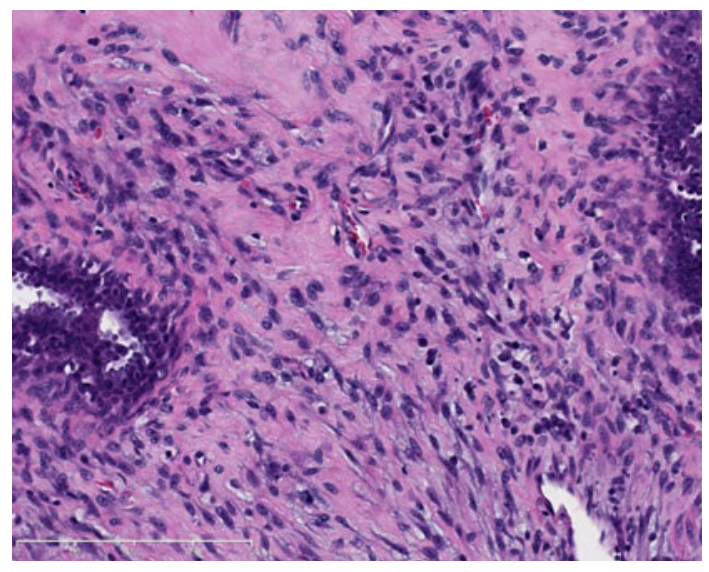

Fig. 6 The microscopic appearance of the removed tumor. The tumor consisted of benign epithelial and stromal cells with no obvious mitosis or cellular atypia. The mass was diagnosed as a benign phyllodes tumor (hematoxylin and eosin staining, magnification $\times 100)$

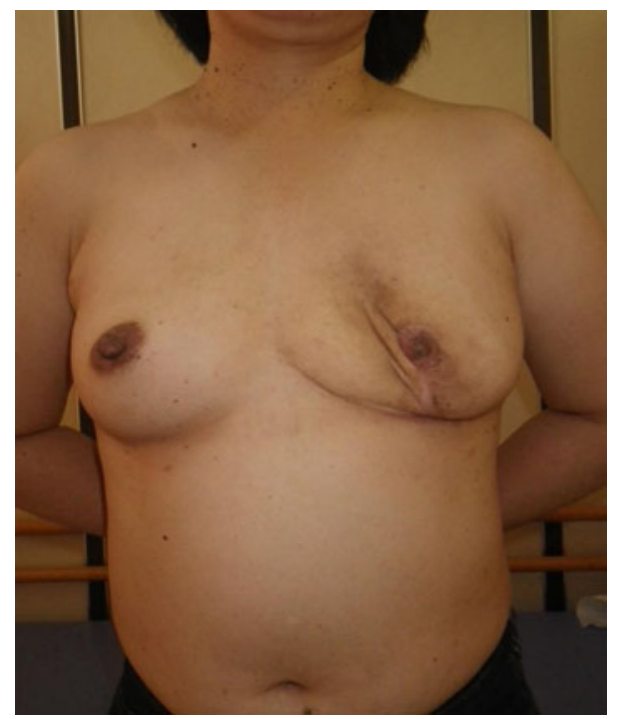

Fig. 7 The postoperative appearance of both breasts 6 months after surgery. Breast symmetry has almost been restored. There are wrinkles at the center of the breast because an insufficient area of skin was removed and because an insufficient volume of glandular tissue remained under the skin nipple-areola complex has been well grafted. However, there were wrinkles at the center of the breast because an insufficient area of skin was removed and because there was an insufficient volume of glandular tissue under the skin (Fig. 7). We had suggested the surgical correction of the ipsilateral breast to her, but she had been satisfied with the cosmetic results with no need for further surgery. Generally speaking, a 6-month period after surgery was not enough for evaluating the cosmetic result. We told the patient that a longer follow-up period would be needed.

\section{Discussion}

In contrast to fibroadenomas, which are considered to be a hyperplastic disease, phyllodes tumors are a neoplastic disease showing a distinct proliferation of breast stromal cells [6]. Although most phyllodes tumors are benign, a few are diagnosed as malignant because of a remarkable proliferation of stromal cells with metastatic potential [7]. Phyllodes tumors sometimes recur in the ipsilateral breast, and therefore, a wide excision with 1-2 cm margins is recommended [7-9]. However, these surgical margins are not realistically possible beneath the overlying skin. In addition, phyllodes tumors originate from the breast stromal cells and hardly invade the skin or nipple-areola complex. From these facts, the adequate length of the surgical margins is not clearly defined [10]. Of course, the total mastectomy may reduce the local recurrence risk of giant phyllodes tumors; however, there have been no controlled randomized trials that compare total and partial mastectomies in terms of disease recurrence. Considering the above, BCS is an alternative option for giant phyllodes tumors. In the case of a high breast volume removed even by the partial mastectomy, oncoplastic surgical techniques are needed.

To correct breast ptosis, a mastopexy reduces the skin and elevates the nipple-areola complex, whereas a reduction mammaplasty comprises a mastopexy and reduction of the parenchymal tissue [11]. There are many skin excision 
patterns for mastopexy and reduction mammaplasty. The best procedure is chosen by evaluating the characteristics of the breast [12-14]. The key to these techniques is the translocation of the nipple-areola. In mastopexy and reduction mammaplasty, the nipple-areola is generally moved with a glandular flap to maintain the blood supply. However, because a reliable pedicle is not always preserved, especially in case of an extremely large breast tissue reduction [14], a technique using a free nipple-areola graft may be indicated [15]. In this case we changed the initial plan for the nipple-areola translocation with a glandular flap. The free grafting of the nipple-areola complex should have been preplanned as an alternative option in this case of a large phyllodes tumor because there is a high possibility of no glandular flap being constructed under the nipple-areola.

In the present case, the inverted-T scar technique was applied. This technique was first reported by Wise et al. [16], and it has become one of the most popular mastopexy and reduction mammaplasty techniques. The skin incision patterns used in this technique enable a wide range of skin and parenchymal tissue reduction. In the present case, a relatively large amount of the expanded skin was removed. However, we could not estimate how the expanded skin would shrink after tumor resection. As a result, there were wrinkles at the center of the breast, likely because the horizontal resection of the skin was insufficient. To achieve better cosmetic results in the case of large phyllodes tumors with expanded skin, the amount of skin resection should be large enough, and in addition the dislocated glandular tissue needs to be moved for a natural breast mount if possible.

In conclusion, giant phyllodes tumors sometimes result in deformity of the conserved breast after BCS. In such cases, mammaplasty using the inverted T-scar technique is considered useful.

Conflict of interest The authors declare that we have no conflict of interest.

\section{References}

1. Berry MG, Fitoussi AD, Curnier A et al (2010) Oncoplastic breast surgery: a review and systematic approach. J Plast Reconstr Aesthet Surg 63:1233-1243

2. Chepla KJ, Armijo BS, Ponsky TA et al (2011) Benefits of immediate dermoglandular preserving reconstruction following giant fibroadenoma excision in two patients. J Plast Reconstr Aesthet Surg 64:244-247

3. Yamamoto Y, Sugihara T (1998) Application of reduction mammaplasty in treatment of giant breast tumour. Br J Plast Surg 51:109-112

4. Yilmaz M, Vayvada H, Menderes A et al (2003) Reduction mammaplasty for phyllodes tumor causing asymmetry in an adolescent female. Breast J 9:426-427

5. Beier JP, Jaeger K, Horch RE (2006) Reduction mammaplasty for benign phyllodes tumour in an adolescent female-a 13-year follow up. Breast 15:550-553

6. Noguchi S, Yokouchi H, Aihara T et al (1995) Progression of fibroadenoma to phyllodes tumor demonstrated by clonal analysis. Cancer 76:1779-1785

7. Jeanne AP (2000) Phyllodes tumors. Diseases of the breast, 3rd edn. Lippincott Williams \& Wilkins, Philadelphia, pp 669-675

8. August DA, Kearney T (2000) Cystosarcoma phyllodes: mastectomy, lumpectomy, or lumpectomy plus irradiation. Surg Oncol 9:49-52

9. Barth RJ Jr (1999) Histologic features predict local recurrence after breast conserving therapy of phyllodes tumors. Breast Cancer Res Treat 57:291-295

10. Jang JH, Choi MY, Lee SK et al (2012) Clinicopathologic risk factors for local recurrence of phyllodes tumors of the breast. Ann Surg Oncol 19:2612-2617

11. Karp NS (2006) Mastopexy and mastopexy augmentation. Grabb \& Smith's plastic surgery, 6th edn. Lippincott Williams \& Wilkins, Philadelphia, pp 585-592

12. Rohrich RJ, Thornton JF, Jakubietz RG et al (2004) The limited scar mastopexy: current concepts and approaches to correct breast ptosis. Plast Reconstr Surg 114:1622-1630

13. Andrades P, Prado A (2008) Understanding modern breast reduction techniques with a simplified approach. J Plast Reconstr Aesthet Surg 61:1284-1293

14. Nahai FR, Nahai F (2008) MOC-PSSM CME article: breast reduction. Plast Reconstr Surg 121:1-13

15. Casas LA, Byun MY, Depoli PA et al (2001) Maximizing breast projection after free-nipple-graft reduction mammaplasty. Plast Reconstr Surg 107:961-964

16. Wise RJ (1956) A preliminary report on a method of planning the mammaplasty. Plast Reconstr Surg 17:367-375 